\title{
SEMIGROUP OF OPERATORS ON DUAL BANACH SPACES
}

\author{
ANTHONY TO-MING LAU ${ }^{1}$
}

\begin{abstract}
In this paper, we give a short and simple proof to a more general version of a recent result of Yeadon for semigroups of weak*-continuous operators on a dual Banach space. Our result has application to amenable groups and property $\mathbf{P}$ of a von Neumann algebra.
\end{abstract}

1. Introduction. Let $E$ be a dual Banach space with a (fixed) predual $E_{*}$, i.e. $E_{*}$ is a Banach space such that $\left(E_{*}\right)^{*}=E$. Let $s$ be a semigroup of linear operators from $E$ into $E$ satisfying:

(1) $\|s x\|=\|x\|$ for all $s \in S$ and $x \in E$.

(2) Each $s \in \mathcal{S}$ is a continuous linear map from $\left(E\right.$, weak $\left.{ }^{*}\right)$ into $(E$, weak $\left.{ }^{*}\right)$.

Let $E^{\mathcal{S}}=\{x \in E ; s(x)=x$ for all $s \in \mathcal{S}\}$ and $K_{x}=$ weak $^{*}$-closure of $\operatorname{co}\{s(x) ; s \in \mathcal{S}\}$ (here co $X$ will denote the convex hull of a subset $X$ of a linear space) for each $x \in E$. Then $E^{\delta}$ is a weak*-closed linear subspace of $E$. Recently, Yeadon [12], ultilizing a method in [9, Lemma 5], proved that if $K_{x} \cap E^{\mathcal{S}}$ is nonempty for each $x \in E$, then there exists a bounded linear operator $P$ from $E$ onto $E^{\S}$ such that $P(x) \in K_{x} \cap E^{\S}$ for each $x \in E$. Furthermore, $P$ commutes with any weak*-continuous linear operator $Q$ commuting with each $s$ in $S$.

In this paper we prove a more general version of Yeadon's result. Our proof is simple and different from that of Yeadon. In $\$ 3$, we concentrate on various applications of our theorems to obtain some of the results on invariant mean in [2], [3], [5], [6], [7], [10] and [11], and on property $\mathrm{P}$ of a von Neumann algebra in [9].

2. The main theorem. Let $\mathscr{T}(E)$ be the space of bounded linear operators from $E$ into $E$. By the weak*-operator topology on $\mathscr{B}(E)$, denoted by $W^{*} O T$, we shall mean the locally convex topology determined by the family of seminorms $\left\{p_{x, \phi} ; x \in E\right.$ and $\left.\phi \in E_{*}\right\}$ where $p_{x, \phi}(T)=|P(T x)|$. As known [4, p. 973], the unit ball of $9(E)$ is compact in the $W^{*} O T$. Let co $\delta$ denote the closure of co $\mathcal{S}$ in the $W^{*} O T$. Then $\left(\overline{\mathrm{co}} \mathcal{S}, W^{*} O T\right)$ is a semigroup and a compact Hausdorff space such that for each $k \in \overline{c o} \delta$, and each $s \in S$, the following mappings from ( $\left.\overline{\mathrm{co}} \delta, W^{*} O T\right)$ into $\left(\overline{\mathrm{co}} \varsigma, W^{*} O T\right)$ are continuous:

Received by the editors January 6, 1975 .

AMS (MOS) subject classifications (1970). Primary 22A25, 43A07, 47D05; Secondary 47C15, 47D20, 47H10.

Key words and phrases. Dual Banach spaces, semigroup of operators, amenable semigroup, stationary, von Neumann algebra, property $P$, invariant means.

${ }^{1}$ This research is supported by NRC Grant A7679. 
(1) $h \rightarrow h k$,

(2) $h \rightarrow s h$.

Let $\mathcal{G} \subseteq \overline{\mathrm{co}} S$ be a closed subsemigroup of $\overline{\mathrm{co}} S$ and let

$$
\mathcal{G}(x)=\{g(x) ; g \in \mathcal{G}\} \text {. }
$$

A subset $X \subseteq E$ is $\mathcal{G}$-invariant if $\mathcal{G}(x) \subseteq X$ for each $x \in X$.

If $F \subseteq E^{\delta}$, an operator $P \in \cup(E)$ is $F$-stationary on $X$ if for each $x \in X$, $P(x) \in F$.

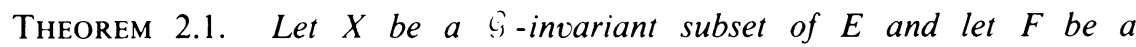
weak ${ }^{*}$-closed subset of $E^{\S}$. If $\mathcal{G}(x) \cap F$ is nonempty for each $x \in X$, then $\mathcal{G}$ contains an $F$-stationary operator on $X$. In this case, $\mathcal{G}(x) \cap F=\{P(x)$; $P \in \mathcal{G}$ and $P$ is $F$-stationary on $X\}$ for each $x \in X$.

Proof. For each $x \in X$, let $\mathscr{H}(x)=\{g \in \mathcal{G} ; g(x) \in F\}$. Then $\mathscr{K}(x)$ is a nonempty closed subset of $\mathcal{G}$. Furthermore, if $\sigma=\left\{x_{1}, \ldots, x_{n}\right\}$ is any finite subset of $X$ and $g \in \cap\left\{\mathcal{H}\left(x_{i}\right) ; i=1, \ldots, n-1\right\}$, pick $g^{\prime} \in \mathcal{G}$ such that $g^{\prime}\left(g\left(x_{n}\right)\right) \in F$. It follows that $\left(g^{\prime} \cdot g\right)\left(x_{i}\right)=g\left(x_{i}\right)$ for all $i=1, \ldots, n-1$ and $g^{\prime} \cdot g \in \cap\left\{\mathfrak{h}\left(x_{i}\right) ; i=1, \ldots, n\right\}$. Consequently, if $x_{0} \in X$ and $z \in$ $\mathcal{G}\left(x_{0}\right) \cap F$, the sets $\mathscr{K}^{\prime}(x)=\left\{g \in \mathcal{Q} ; g(x) \in F\right.$ and $\left.g\left(x_{0}\right)=z\right\}$ are closed in $\mathcal{G}$ and have finite intersection property. By compactness, $\cap\left\{\mathcal{H}^{\prime}(x) ; x \in X\right\}$ is nonempty, and any $P$ in this intersection is $F$-stationary and $P\left(x_{0}\right)=z$.

REMARK. If $\mathcal{G}=\overline{\mathrm{co}} \mathcal{S}$ and $E^{\varsigma}=F$, then $\mathcal{G}(x)=$ weak ${ }^{*}$-closure of $\operatorname{co}\{s x$; $s \in \delta\}$. Hence, the first part of Theorem 2.1 yields the proposition in [12].

\section{Applications.}

A. Invariant means on discrete semigroups. Let $S$ be a semigroup and $l_{\infty}(S)$ be the Banach space of bounded real-valued (or complex-valued) functions on $S$ with supremum norm. For each $a \in S$, define the left and right translation operators on $l_{\infty}(S)$ by $\left(l_{a} f\right)(t)=f(a s)$ and $\left(r_{a} f\right)(s)=f(s a)$ for all $s \in S, f \in l_{\infty}(S)$. Let $X$ be a closed translation invariant linear subspace of $l_{\infty}(S)$ containing constant one function 1. A linear functional $\phi$ on $X$ is called a mean if $\|\phi\|=1$ and $\phi \geqslant 0$. A mean on $X$ is called a left invariant mean if $\phi\left(l_{a} f\right)=\phi(f)$ for all $a \in S$ and all $f \in X$ (see Day [1]).

The subspace $X$ is called left introverted [respectively, left $m$-introverted] if for each mean [respectively, multiplicative mean] $\phi$ on $l_{\infty}(S)$, the function $s \rightarrow \phi\left(l_{s} f\right)$ is in $X$. (See [1, p. 540] and [8, p. 121].)

Let $S=\left\{r_{a} ; a \in S\right\}$. Since the weak*-topology and the topology of pointwise convergence agree on bounded subsets of $l_{\infty}(S)$, it follows from [3, Lemma 2 and the proposition following] that $X$ is left introverted [respectively, left $m$-introverted] if and only if $X$ is $\overline{c o} \bar{s}$ - [respectively, $\bar{\varsigma}$-] invariant.

THEOREM 3.1 (Granirer and Lau [3]). If $X$ is left introverted and for each $f \in X, \overline{\mathrm{co}} \delta(f)$ contains a constant function, then $X$ has a left invariant mean. In this case for each $f \in X, \alpha \cdot 1 \in \overline{\mathrm{co}} \mathrm{s}(f)$ if and only if $m(f)=\alpha$ for some left invariant mean $m$ on $X$.

Proof. Let $F$ be the one-dimensional subspace of $l_{\infty}(S)$ consisting of constant functions. If $P \in \overline{\mathrm{co}} \varsigma$ is $F$-stationary on $X$, define $m(f)=(P f)(a)$ 
for some fixed $a \in S$. Since left and right translations commute, $m$ is a left invariant mean on $X$. Now use Theorem 2.1.

Similarly we have

THEOREM 3.2 (Granirer and Lau [3]). If $X$ is a left $m$-introverted subalgebra of $l_{\infty}(S)$ and for each $f \in X, \bar{S}(f)$ contains a constant function, then $X$ has a multiplicative left invariant mean. In this case for each $f \in X, \lambda \cdot 1 \in \bar{s}(f)$ if and only if $m(f)=\lambda$ for some multiplicative left invariant mean $m$ on $X$.

REMARK. 1. Theorem 3.1 is proved by Mitchell [5, Theorem 3] for the case $X=l_{\infty}(S)$ and Theorem 3.2 is proved by Granirer [2, Theorem 1] for the case $X=l_{\infty}(S)$. Their proofs are completely different.

2 (Granirer and Lau [3]). If $X$ is introverted and for each $f \in X$ and each $a \in S, \overline{\mathrm{co}} \mathcal{S}\left(f-l_{a} f\right)$ contains the zero function, then $X$ has a left invariant mean. To see this, let $F=\{0\}$. Apply Theorem 2.1 ; we obtain $T \in \overline{\text { co }} \varsigma$ such that $T\left(f-l_{a} f\right)=0$ for all $f \in X$ and $a \in S$.

3. If for each $A \subseteq S, \bar{S}\left(1_{A}\right)$ contains either the zero function or the one function, then $l_{\infty}(S)$ has a multiplicative left invariant mean. Indeed, let $F=\{0,1\}$. By Theorem 2.1, there exists $T \in \bar{S}$ such that $T\left(1_{A}\right) \in F$ for all $A \subseteq S$. Then $T$ defines a multiplicative left invariant mean on $l_{\infty}(S)$.

Note that $\bar{\delta}\left(1_{A}\right)$ contains the one function [respectively, the zero function], if and only if $A$ [respectively, $S-A$ ] is left thick (see [7, p. 256] for definition). Hence, this yields [6, Theorem 1(f) $\rightarrow$ (a)] and [5, Theorem 3].

B. Invariant means on locally compact groups. Let $G$ be a locally compact group with a fixed left Haar measure $\lambda$. Let $P(G)=\left\{\phi \in L_{1}(G) ;\|\phi\|=1\right.$ and $\phi \geqslant 0\}$. For each $\phi \in P(G)$, define the translation operators on $L_{\infty}(G)$ by $l_{\phi}(f)=\phi * f$ and $r_{\phi}(f)=f * \tilde{\phi}$ for all $f \in L_{\infty}(G)$. Let $X$ be a closed linear subspace of $L_{\infty}(G)$ which is invariant under $r_{\phi}$ and $l_{\phi}$ for each $\phi \in P(G)$ and containing constants. An element $m \in X^{*}$ is called a mean if $\|m\|=1$ and $m \geqslant 0$. A mean $m$ on $X$ is called a topological left invariant mean if $m\left(l_{\phi} f\right)$ $=m(f)$ for each $\phi \in P(G)$ and each $f \in X$.

Let $\delta=\left\{r_{\phi} ; \phi \in P(G)\right\}$. Then $\delta$ is a semigroup of weak*-continuous contractions on $L_{\infty}(G)$. Furthermore, $X$ is $\bar{\delta}$-invariant (note that $\delta=$ co $\delta$ ) if and only if $X$ is topologically left introverted, i.e. for each mean $m$ on $L_{\infty}(G)$ and each $f \in X$, the functional $\phi \rightarrow m\left(\Delta^{-1} \tilde{\phi} * f\right)$ defined on $L_{1}(G)$ is

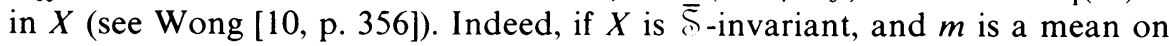
$L_{\infty}(G)$, let $\left\{\phi_{\alpha}\right\}$ be a net in $P(G)$ such that $\phi_{\alpha}$ converges to $m$ in the weak*-topology. By compactness of $\overline{\mathcal{S}}$, we may assume that the net $\left\{r_{\phi_{\alpha}}\right\}$ converges to some $T \in \bar{S}$ in the $W^{*} O T$. Hence, for each $\phi \in L_{1}(G)$,

$$
\begin{aligned}
m\left(\Delta^{-1} \tilde{\phi} * f\right) & =\lim _{\alpha} \phi_{\alpha}\left(\Delta^{-1} \tilde{\phi} * f\right) \\
& =\lim _{\alpha} \phi\left(f * \tilde{\phi}_{\alpha}\right) \quad(\text { see }[10, \text { Lemma 3.1(c) }]) \\
& =\lim _{\alpha} \phi\left(r_{\phi_{\alpha}} f\right)=\phi(T f) .
\end{aligned}
$$

Hence, $X$ is topologically left introverted. The other direction can be proved similarly.

ThEOREM (Wong [10, Theorem 5.4]). Assume that $X$ is topologically left 
introverted. If for each $f \in X, \bar{S}(f)$ contains a constant function, then $X$ has a topological left invariant mean. In this case for each $f \in X, \beta \cdot 1 \in \bar{\delta}(f)$ if and only if there is a topological left invariant mean $m$ on $X$ such that $m(f)=\beta$.

Proof. Apply Theorem 2.1 with $F$ being the one-dimensional subspace of $F$ consisting of the constant functions.

REMARK. Recently, Wong [11, Theorem $3.1(1) \Rightarrow(4)$ ] proved a similar theorem for locally compact topological semigroups. It is easy to see that this result is also a consequence of Theorem 2.1. We omit the details.

C. von Neumann algebras with property $\mathrm{P}$. Let $M$ be a von Neumann algebra acting on a Hilbert space $H$ and let $M^{\prime}$ be the commutant of $M$. Assume that $M$ contains the identity operator on $H$. Let $M^{u}$ be the group of unitary elements in $M$. For each $u \in M^{u}$, define $T_{u}(x)=u^{*} x u$ for each $x \in \Re(H)$. Let $\delta=\left\{T_{u} ; u \in M^{u}\right\}$. Then $M$ is said to have property $\mathrm{P}$ if for each $x \in \mathscr{B}(H) \overline{c o} \varsigma(x) \cap M^{\prime}$ is nonempty. It is easy to see that the following slight improvement of Schwartz's result [9, Lemma 5] is also a consequence of Theorem 2.1.

THEOREM. Let $\mathcal{G}$ be a closed subsemigroup of $\overline{\mathrm{co}} \S$ containing $\$$. If $\mathcal{G}(x) \cap M^{\prime} \neq \varnothing$ for each $x \in \mathscr{G}(H)$, there exist $P \in \mathcal{G}$ such that

$$
P(x) \in M^{\prime} \text { for each } x \in \mathscr{G}(H) \text {. }
$$

In this case, for each $x \in \mathscr{G}(H), z \in \mathcal{G}(x) \cap M^{\prime}$ if and only if there exist $P \in \mathcal{G}$ satisfying $(*)$ and $P(x)=z$.

\section{REFERENCES}

1. M. M. Day, Amenable semigroups, Illinois J. Math. 1(1957), 509-544. MR19, 1067.

2. E. Granirer, Extremely amenable semigroups. II, Math Scand. 20(1967), 93-113. MR35 \#3422.

3. E. Granirer and A. T. Lau, Invariant means on locally compact groups, Illinois J. Math. 15(1971), 249-257. MR43 \#3400.

4. Richard V. Kadison, The trace in finite operator algebras, Proc. Amer. Math. Soc. 12(1961), 973-977. MR25 \#3385.

5. A. T. Lau, Extremely amenable algebras, Pacific J. Math. 33(1970), 329-336. MR41 \# 7005.

6. T. Mitchell, Fixed points and multiplicative left invariant means, Trans. Amer. Math. Soc. 122(1966), 195-202. MR32 \#7662.

7. Constant functions and left invariant means on semigroups, Trans. Amer. Math. Soc. 119(1965), 244-261. MR33 \#1743.

8. $\quad$ Function algebras, means and fixed points, Trans. Amer. Math. Soc. 130(1968), 117-126. MR36 \#666.

9. J. T. Schwartz, Two finite, non-hyperfinite, non-isomorphic factors, Comm. Pure Appl. Math. 16(1963), 19-26. MR26 \#6812.

10. James C. S. Wong, Topologically stationary locally compact groups and amenability, Trans. Amer. Math. Soc. 144(1969), 351-363. MR40 \#2781.

11. , An ergodic property of locally compact amenable semigroups, Pacific J. Math. 48(1973), 615-619.

12. F. Y. Yeadon, Fixed points and amenability: a counterexample, J. Math. Anal. Appl. 45(1974), 718-720.

Department of Mathematics, University of Alberta, Edmonton, Alberta, Canada 